\title{
Robust Packet Detector based Automatic Gain Control Algorithm for OFDM-based Ultra-WideBand systems
}

\author{
Chun-Yuan Chu, Jyh-Ting (Justin) Lai*, and An-Yeu (Andy) Wu \\ Graduate Institute of Electronics Engineering, and Department of Electrical Engineering, \\ National Taiwan University \\ *Sitronix Technology Corporation
}

\begin{abstract}
We propose a robust auto gain control (AGC) algorithm for OFDM-based ultra-wideband (UWB) system. In the UWB system, the transmitted signal power is limited to -41.25 $\mathrm{dBm}$ in a $1-\mathrm{MHz}$ bandwidth. Therefore, it is difficult for front-end receiver operation by using traditional power estimation approach. Based on the proposed packet detector (PD), the signal power estimation is much more accurate. On the other hand, saturation of the ADC output is another critical issue. We provide a robust AGC convergence mechanism solving this problem. After the AGC operation converges, the received signal power is within the error margin relative to target power. This power error is less than $0.5 \mathrm{~dB}$.
\end{abstract}

\section{INTRODUCTION}

Multi-Band Orthogonal Frequency-Division Multiplexing (MB-OFDM) technology has been applied to wireless personal area networks [1]. This technique combines OFDM with frequency hopping, and it increases both the traffic capacity and the frequency diversity. It follows the relevant Federal Communications Commission (FCC) regulations, which specify that the $7500 \mathrm{MHz}$ spectrum from $3.1 \mathrm{GHz}$ to $10.6 \mathrm{GHz}$. The large bandwidth enables a demanding throughput and makes high quality wireless multimedia application possible.

OFDM system is sensitive to transmitter/receiver offset, such as carrier frequency offset (CFO) and sample frequency offset (SFO). Therefore, an accurate synchronization is essential. In general, AGC is one of urgent initialization functions because of its critical influence on received SNR. AGC inaccuracy will degrade the performance of all subsequent operations including time/frequency synchronization, channel estimation, etc. However, there are other wireless services in thespectrum for UWB, such as [2].

Financial support from NSC (grant no. NSC 95-2219-E-002-020) is greatly appreciated.

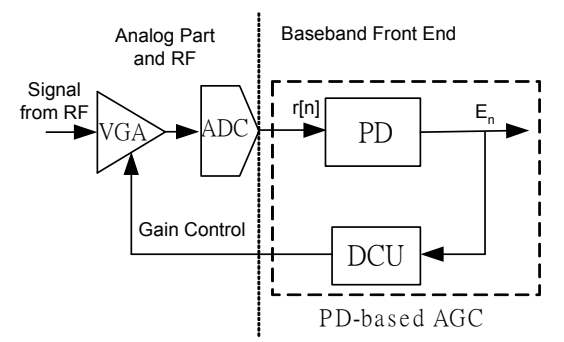

Fig. 1 Block diagram of the AGC system.

Therefore, the power spectral density of MB-OFDM must not exceed $-41.25 \mathrm{dBm}$ in a $1-\mathrm{MHz}$ bandwidth [1] to avoid interfering with existing services. The worst received SNR is $-8.4 \mathrm{~dB}$. In this condition, the power component of the received signal would be from noise rather than from the signal itself. It is difficult for front-end receiver operation including AGC and PD.

Fig. 1 is the detailed block diagram of the AGC in our MB-OFDM system. The AGC should converge within the period of Packet Preamble. Because of the feedback control, the iteration bound will also limit the AGC operation speed and result. From the system requirement, the gain error of AGC of the receiver is less than $1 \mathrm{~dB}$. It also increases the design difficulties. To decrease the hardware cost, we can not use the Received Signal Strength Indication (RSSI) to indicate the input strength, the signal information is only from the output of ADC. However, large power/amplitude of input signal results in the saturation of the ADC, so the estimation of input power will be an important issue.

We have proposed an robust PD for UWB system in [3][4]. The SNR of received preamble signal can be enhanced by the proposed PD. In this work, we propose a robust AGC algorithm based on the PD. The PD calculates the power of the received signal accurately even in low SNR. We can utilize the power component from the PD. Besides, the proposed AGC convergence mechanism can cope with the saturation of the ADC. Even in the condition of $\mathrm{SNR}=-10 \mathrm{~dB}$, the error relative to target power is only $0.5 \mathrm{~dB}$. 
This paper is organized as follows. Section 2 provides a brief review of the robust PD and the signal features related to AGC operation. The proposed AGC algorithm is presented in section 3. Section 4 is the simulation results. Section 5 gives a conclusion of this paper.

\section{THE ROBUST PACKET DETECTOR}

The proposed AGC algorithm is aided by the robust PD. In this section, we will review the PD [2]. It comprises 2 subcomponents: symbol combiner (SC), symbol detector $(S D)$. Fig. 2 is the architecture of the robust PD.

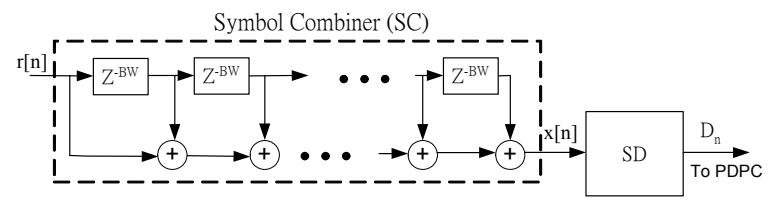

(a)

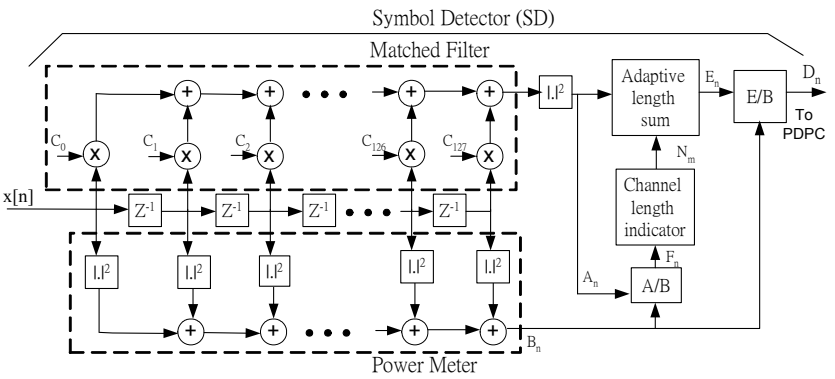

(b)

Fig.2 Datapath block diagrams of the BT-PD: (a) The SC and SD in the PD for $T F C=1$; (b) detailed circuit of the SD.

\subsection{Symbol Combiner}

In order to increase the noise immunity, we use an SC to combine several preamble symbols - instead of using only one symbol - to detect the packet arrival. Because the preamble symbols are repeated, this technique will increase the effective SNR. However, MB-OFDM employs several types of time-frequency code (TFC) in the preamble part to easily identify the packet properties and frequency-hopping sequences. The RF circuit for receiving is fixed at a specific band before the PD formally declares that a packet has arrived, the received signal only appears in the time slot when the transmitted band is the same as the (fixed) receiving band. Therefore, we have to collect signal samples in a queue and sum the suitable time slot when there are signals. Eq.(1) is an example for $\mathrm{TFC}=1,2$ [1]:

$$
x(n)=\sum_{k=0}^{J-1} r(n-B W k), \quad(\text { for } T F C=1,2)
$$

where $r(n)$ is the received baseband signal, $J$ is the number of combined symbols, $B$ is the total number of bands in the band group, $W$ is the total number of samples in one OFDM symbol, and

$$
W=N+L,
$$

where $N$ is the FFT window size and $L$ is the zero-padding duration. Typical values of $B, N$, and $L$ are 3, 128, and 37, respectively. From Eq.(1), the received SNR is enhanced by $J$ times.

\subsection{Symbol Detector}

The SD structure is shown in Fig.2 (b). The upper block in the figure is a matched filter, which is a FIR filter with preamble coefficients as filter coefficients. We utilize the matched filter output to estimate the received signal power. Before the power estimation approach, we define some important signal vectors as

$$
\begin{gathered}
\mathbf{c} \equiv\left[c_{0}, c_{1}, \cdots, c_{N-1}\right]^{\mathrm{T}}, \\
\mathbf{h}=\left[h_{0}, h_{1}, h_{2}, \cdots, h_{N_{m}-1}\right]^{\mathrm{T}}, \\
\mathbf{s}(n)=[s(n), s(n+1), \cdots, s(n+W-1)]^{\mathrm{T}}, \\
s(n)=\left\{\begin{array}{cc}
c_{((n))_{W}}, \text { if }((n))_{W}<N \\
0, & \text { otherwise }
\end{array}\right. \\
\mathbf{S}(n)=\left[\mathbf{s}(n), \mathbf{s}(n-1), \cdots, \mathbf{s}\left(n-N_{m}+1\right)\right]^{\mathrm{T}}, \\
\mathbf{x}(n)=J \mathbf{S}(n) \mathbf{h}+\sum_{k=0}^{J-1} \mathbf{n}_{k}(n), \\
\mathbf{x}(n)=[x(n), x(n+1), \cdots, x(n+W-1)]^{\mathrm{T}},
\end{gathered}
$$

where the $c_{i}$ variables are the preamble coefficients, $\left\{h_{l}\right\}$ denotes the normalized CIR, $N_{m}$ is the channel duration, $\mathbf{s}(n)$ is the transmitted signal vector, $\mathbf{x}(n)$ is the received signal vector, $((n))_{W}$ denotes the remainder of $n$ divided by $W$, and $\mathbf{n}_{k}(n)$ is the identical independent distribution (i.i.d.) noise vector with power $\sigma_{n}^{2}$. The output power $A_{n}$ of the matched filter is

$$
A_{n}=\mathbf{c}^{H} \mathbf{x}(n) \mathbf{x}^{H}(n) \mathbf{c} .
$$

Taking the expectation value of Eq.(9) yields

$$
\begin{gathered}
\widetilde{A}_{n} \equiv E\left[A_{n}\right]=\mathbf{C}^{H} E\left[\mathbf{X}(n) \mathbf{X}^{H}(n)\right] \mathbf{C} \\
=J^{2} N^{2} \sigma_{s}^{2}\left|\hat{h}_{\kappa}\right|^{2}+J N \sigma_{n}^{2}
\end{gathered}
$$

Therefore, the expectation value of the adaptive length sum output is

$$
\widetilde{E}_{n} \equiv E\left[E_{n}\right]=\sum_{k=n}^{n+N_{m}-1} \widetilde{A}_{k}=J^{2} N^{2} \sigma_{s}^{2}+J N_{m} N \sigma_{n}^{2},
$$

where we use the property of the normalized channel, that is,

$$
\sum_{\kappa=0}^{N_{m}-1}\left|h_{\kappa}\right|^{2}=1
$$

and assume that $N_{m}$ is known and can be obtained from the CLI. From Eq.(11), the input power is expressed as

$$
\sigma_{s}^{2}=\frac{\hat{E}_{n}-J N N_{m} \sigma_{n}^{2}}{J^{2} N^{2}},
$$

where $\hat{E}_{n}$ is the actual output of the adaptive length sum.

\section{PD-BASED AGC ALGORITHM}

\subsection{Target Received Power}


The peak-average-power-ratio (PAPR) issue of traditional OFDM system is often based on the higher SNR environment. The target received power $P_{T}$ is as

$$
P_{T}=\frac{Y^{2}}{P A P R} \text {. }
$$

where $Y$ is the half swing of ADC. Because the SNR would be very low in the MB-OFDM system, we need to use the signal $\hat{E}_{n}$ from the PD to estimate the power of input signal and thus adjust the gain of VGA. The estimated power is

$$
\hat{\sigma}_{s}^{2}=\frac{\hat{E}_{n}}{J^{2} N^{2}},
$$

\subsection{Gain Update Mechanism}

The input dynamic range of MB-OFDM system will be as large as $26 \mathrm{~dB}$. Hence, the $\mathrm{ADC}$ may saturate as the received signal power is too large. Fig. 3 shows the relation between input and output signal of the ADC. We separate the input power into three regions: linear region, week saturation region, and strong saturation region. If the power of the ADC input is in the linear region, we can calculate the input power accurately. However, if the power of the ADC input is in the strong saturation region, it is very hard to estimate the input signal with accuracy.

At first, we provide the derivation the convergence mechanism in linear region. Assume the symbol index is $m$, the current gain of VGA is $G(m)$, and the received signal power is $P_{R}$, so the amplified received power is

$$
P(m)=P_{R} G^{2}(m),
$$

Because we expect the power of the ADC input is exactly the target power in next symbol, we have following equation

$$
P_{T}=P_{R} G^{2}(m+1),
$$

where $P_{T}$ is the target converged power. From Eq. (17) and Eq. (18), we can obtain the gain updating equation

$$
G(m+1)=\sqrt{P_{T} / P(m)} \cdot G(m), P(m)<P_{\text {Saturation }}
$$

where $P_{\text {Saturation }}$ denotes the boundary power between linear and saturation level for the ADC input. Therefore, we can utilize Eq. (19) to calculate the exact gain to next OFDM symbol in order to get the optimal amplified signal power. The AGC can converge easily in the linear region.

After we obtain gain update mechanism in the linear region, we have to consider the saturation region. As mentioned above, we can only estimate the received signal power from the output of the ADC, we can not get a correct estimation in the saturation region. Therefore, we do not use the estimated power for updating. The propose the gain update method by the iterative adjusting as follows

$$
G(m+1)=\sqrt[k]{G(m)}, P(m) \geq P_{\text {Saturation }},
$$

where $\mathrm{k}$ is the convergence factor. The convergence rate is faster if the $\mathrm{k}$ is larger, however, the larger $\mathrm{k}$ result in the $\mathrm{P}(\mathrm{m}+1)$ too small to correctly receiving even though the $\mathrm{P}(\mathrm{m}+1)$ is in linear region. Finally, the gain will stop to update if the received signal power is within the error margin relative to target power $P_{T}$.

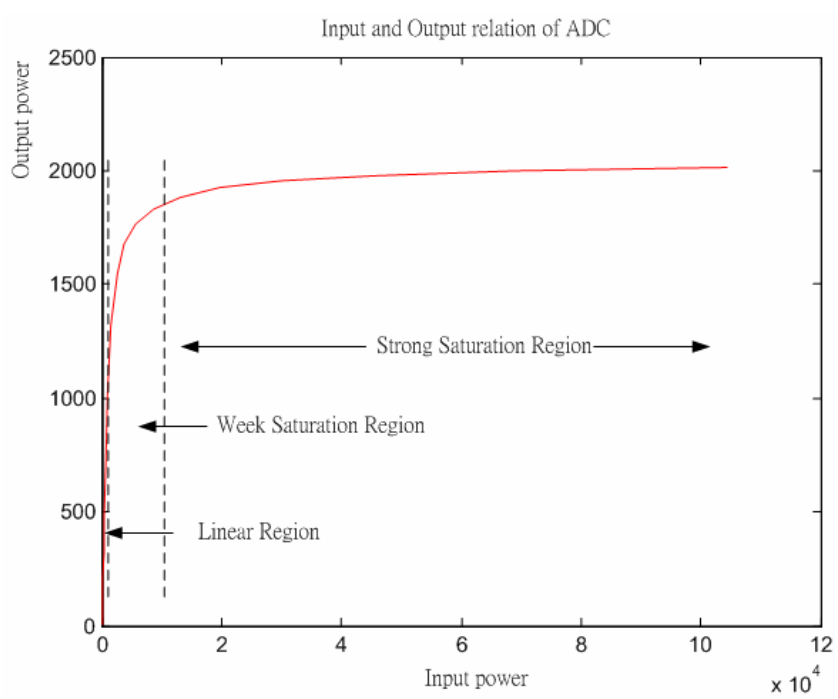

(a)

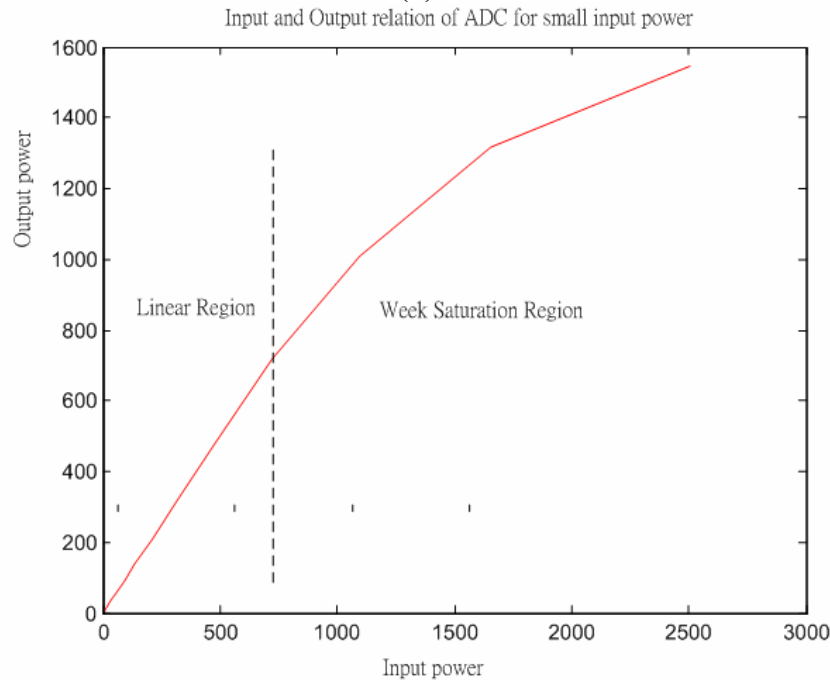

(b)

Fig.3 The relation between input and output signal of ADC.for (a) full scale (b) Zoom-in curve for small input power.

\section{SIMULATION RESULTS}

In this section, we show the simulation results to verify the proposed PD-based AGC algorithm. First, we define the power estimation error as

$$
\text { error }=10 \cdot \log _{10} \frac{\sigma_{s}^{2}}{\hat{\sigma}_{s}^{2}},
$$

where $\sigma_{s}^{2}$ is the actual signal power, and $\hat{\sigma}_{s}^{2}$ is defined in Eq. (16). Fig.4 is the power estimation error. From Fig.4, it shows that the power estimation error is only $0.5 \mathrm{~dB}$ in the PD-based AGC algorithm, even at $\mathrm{SNR}=-10 \mathrm{~dB}$. By 
traditional approach, the performance degradation is huge in low SNR.

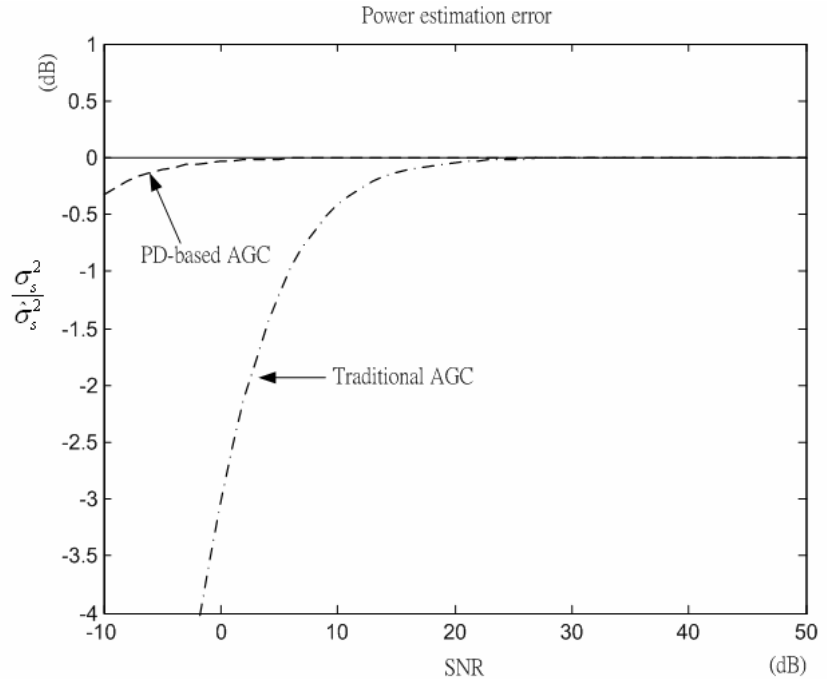

Fig.4 The power estimation error

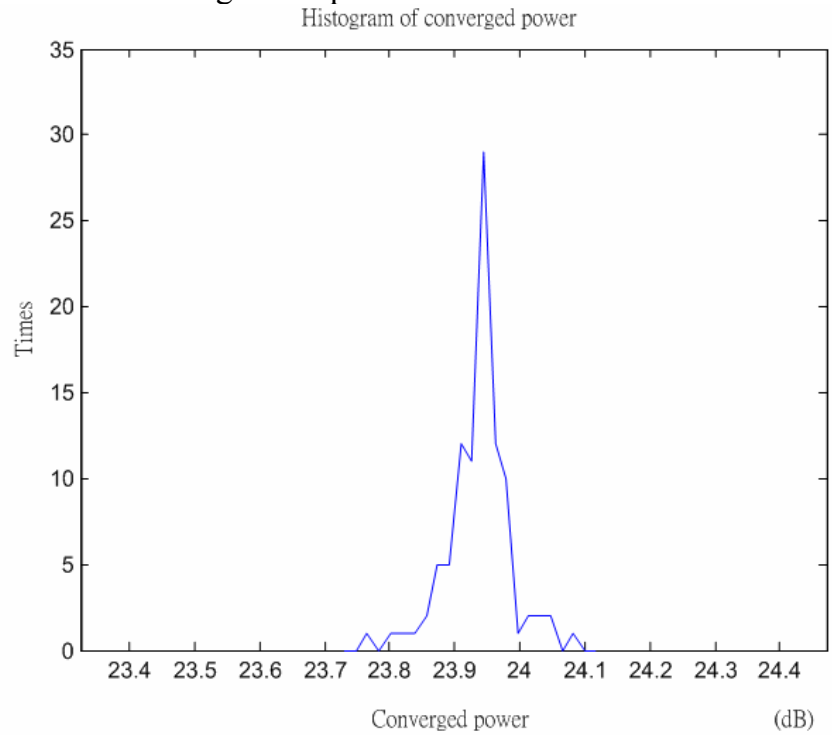

Fig.5 The histogram of converged power of the PD-based AGC.

Fig. 5 is the histogram of finial converged power for the channel with $15 \mathrm{~ns}$ delay spread. From Fig.5, the variance of the final converged power is also $0.5 \mathrm{~dB}$.

\section{CONCLUSION}

In MB-OFDM system, it is very difficult to estimate received signal power. Hence, the traditional AGC can not operate accurately. We propose a robust AGC algorithm that can cope with this problem. This AGC algorithm is based on the robust PD we proposed before. The PD serves as a power estimator, and the estimation error is only $0.5 \mathrm{~dB}$ when $\mathrm{SNR}=-10 \mathrm{~dB}$. Besides, our AGC convergence mechanism is tolerant to the saturation of the ADC.

\section{REFERENCE}

[1] "Multiband OFDM physical layer proposal for IEEE 802.15 Task Group 3a,’ Sept. 14, 2004.

[2] IEEE 802.11a Standard, ISO/IEC 8802-11: 1999/Amd $1: 2000(\mathrm{E})$.

[3] Jyh-Ting Lai, Chun-Yuan Chu, An-Yeu Wu, and WenChiang Chen, "A Robust Band-Tracking Packet Detector (BT-PD) in OFDM-Based Ultra-WideBand Systems," in Proc. IEEE Workshop on Signal Processing Systems (SiPS2006), Banff, Canada, pp. 165-170, Oct. 2006

[4] Jyh-Ting Lai, Chun-Yuan Chu, An-Yeu Wu, and WenChiang Chen, "A Low Cost Packet Detector in OFDMbased Ultra-WideBand systems," in Proc. IEEE Workshop on Signal Processing Systems (SiPS-2006), Banff, Canada, pp. 171-176, Oct. 2006. 